\title{
ORIGINAL ARTICLE Contribution of a first-degree family history of diabetes to increased serum adipocyte fatty acid binding protein levels independent of body fat content and distribution
}

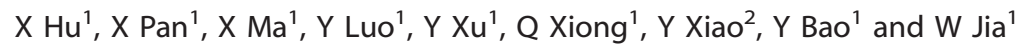

\begin{abstract}
BACKGROUND/OBJECTIVES: First-degree relatives of patients with diabetes bear an increased risk of diabetes, overweight/obesity and cardiovascular disease. Accumulating evidence indicates that circulating concentrations of adipokines are altered in individuals with a first-degree family history of diabetes (FHD), but the adipokine adipocyte fatty acid binding protein (A-FABP) has been rarely studied in this population. The present study explored the association between a first-degree FHD and serum A-FABP levels. SUBJECTS/METHODS: A total of 1962 normoglycemic participants were divided into subgroups of men, premenopausal women and postmenopausal women. Serum A-FABP levels were measured using a sandwich enzyme-linked immunoabsorbent assay. Abdominal fat distribution, including visceral fat area and subcutaneous fat area, was assessed by magnetic resonance imaging. RESULTS: Totals of 792 men, 544 premenopausal women and 626 postmenopausal women were enrolled. Serum A-FABP levels were much higher in subjects with a first-degree FHD than in those without an FHD in all subgroups (all $P<0.05$ ). Logistic regression analysis revealed an independent and positive relationship between a first-degree FHD and serum A-FABP levels in men $(P=0.029)$, premenopausal women $(P=0.036)$ and postmenopausal women $(P=0.008)$. Multiple stepwise regression analysis showed that a first-degree FHD was an independent factor positively associated with serum A-FABP levels in men (standardized $\beta=0.068, P=0.029$ ), premenopausal women (standardized $\beta=0.090, P=0.018$ ) and postmenopausal women (standardized $\beta=0.102, P=0.004)$.

CONCLUSIONS: Serum A-FABP levels were increased significantly in normoglycemic individuals with a first-degree FHD. The contribution of the first-degree FHD to the elevated serum A-FABP levels was independent of total body fat content and abdominal fat distribution. Thus, use of serum A-FABP as a biomarker in the first-degree relatives of patients with diabetes may result in overestimation of the risk of obesity-induced metabolic disease and cardiovascular disease.
\end{abstract}

International Journal of Obesity (2016) 40, 1649-1654; doi:10.1038/ijo.2016.147

\section{INTRODUCTION}

A family history of type 2 diabetes mellitus (T2DM) predisposes individuals to developing the diabetes, ${ }^{1-3}$ with first-degree relatives of patients with T2DM having a $30-70 \%$ increased risk of developing the disease. ${ }^{4}$ Both insulin resistance and $\beta$-cell dysfunction have been identified in individuals with a first-degree family history of diabetes (FHD), even in the absence of diabetes. ${ }^{5}$ In addition, heritability of diabetes is suggested to be associated with overweight/obesity, as is an increased susceptibility to cardiovascular disease (CVD)., ${ }^{4,6}$

Accumulating evidence indicates significant differences in the circulating concentrations of adipokines such as adiponectin, omentin-1, visfatin and retinol-binding protein- 4 between individuals with and without an FHD. ${ }^{7-9}$ The adipokines act as a bridge connecting energy homeostasis, immunity, neuroendocrine function, atherosclerosis, T2DM and insulin resistance. ${ }^{7}$ One such adipokine, adipocyte fatty acid binding protein ( $A-F A B P$, also referred to as fatty acid binding protein 4 and adipocyte protein 2 ), accounts for more than $1 \%$ of the total protein secreted from mature adipocytes, and the mRNA and protein expression of A-FABP are considered biomarkers of adipogenic differentiation. ${ }^{10} \mathrm{Xu}$ et al. ${ }^{11}$ proposed A-FABP as a central factor linking obesity and metabolic syndrome in their first description of circulating A-FABP. Subsequent studies confirmed the important role of A-FABP in obesity-related insulin resistance. ${ }^{12}$ Our previous studies suggested that serum A-FABP levels could not only indicate the presence of the subclinical atherosclerosis in Chinese women with normal glucose tolerance, ${ }^{13}$ but also partially induced cardiac injury under diabetic conditions. ${ }^{14}$ Hence, it is important to understand the changes in serum A-FABP levels and the related factors among individuals with a first-degree FHD, specifically as related to accurate evaluation of risk of metabolic disturbance and CVD. To date, however, no data have been published that demonstrate whether serum A-FABP levels are altered in individuals with a first-degree FHD.

Therefore, the goal of the present study was to explore the association of serum A-FABP levels with a first-degree FHD, as well as with related affecting factors. All of the participants enrolled in this study were normoglycemic, for the purpose of attenuating the impact of abnormal glucose metabolism on serum A-FABP levels.

\footnotetext{
'Department of Endocrinology and Metabolism, Shanghai Jiao Tong University Affiliated Sixth People's Hospital, Shanghai Clinical Center for Diabetes, Shanghai Key Clinical Center for Metabolic Disease, Shanghai Diabetes Institute, Shanghai Key Laboratory of Diabetes Mellitus, Shanghai, China and ${ }^{2}$ Department of Radiology, Shanghai Jiao Tong University Affiliated Sixth People's Hospital, Shanghai, China. Correspondence: Dr X Ma and Professor Y Bao, Department of Endocrinology and Metabolism, Shanghai Jiao Tong University Affiliated Sixth People's Hospital, 600 Yishan Road, Shanghai 200233, China.

E-mail: maxiaojing@sjtu.edu.cn or yqbao@sjtu.edu.cn

Received 9 April 2016; revised 24 June 2016; accepted 22 July 2016; accepted article preview online 18 August 2016; advance online publication, 13 September 2016
} 


\section{MATERIALS AND METHODS}

Subjects

The participants of this study were a subgroup of normoglycemic participants with complete clinical data from the Shanghai Obesity Study cohort. ${ }^{15}$ Based on the 1999 World Health Organization criteria, ${ }^{16}$ individuals with impaired glucose regulation or diabetes were not included in the present study. Exclusion of individuals with liver or renal dysfunction, hyperthyroidism or hypothyroidism, acute infection, psychiatric disease, tumors, a history of CVD, current antihypertensive therapy, current lipid-lowering therapy and current replacement therapy with systemic corticosteroids gave a final sample size of 1962 .

A first-degree FHD was defined as having one or more first-degree relatives with diabetes (parent, sibling or offspring). ${ }^{4}$ Owing to gender differences in serum A-FABP levels observed in our previous study, ${ }^{17}$ the present study divided the study population into subgroups of men, premenopausal women and postmenopausal women for analysis.

The Ethics Committee of Shanghai Jiao Tong University Affiliated Sixth People's Hospital preapproved this study, and all participants provided written informed consent prior to participation.

\section{Anthropometric and biochemical assessments}

Body weight, height, waist circumference (W) and resting blood pressure (BP) were measured by standard techniques. ${ }^{15}$ Body mass index (BMI) was calculated as follows: $\mathrm{BMI}=$ weight $(\mathrm{kg}) /$ height $^{2}\left(\mathrm{~m}^{2}\right)$.

The following biochemical indices were measured in morning fasting blood samples: fasting plasma glucose (FPG), glycated hemoglobin $(\mathrm{HbA} 1 \mathrm{c})$, fasting serum insulin (FINS), serum lipid profiles (total cholesterol (TC), triglyceride (TG), high-density lipoprotein cholesterol (HDL-C) and lowdensity lipoprotein cholesterol (LDL-c)) and C-reactive protein (CRP). Approximately $2 \mathrm{~h}$ after eating breakfast, 2-h plasma glucose levels were assessed. Standard laboratory measurements were performed as described previously. ${ }^{15} \mathrm{~A}$ sandwich enzyme-linked immunoabsorbent assay (Antibody and Immunoassay Services, The University of Hong Kong, Hong Kong) was used to determine serum A-FABP levels, and the corresponding intra- and inter-assay coefficients of variation were 6.6 and $8.7 \%$, respectively. The insulin resistance index (homeostasis model assessment-insulin resistance $(\mathrm{HOMA}-\mathrm{IR}))^{18}$ was calculated as follows: HOMA-IR $=$ FINS $\left(\mathrm{mU} \mathrm{I}^{-1}\right) \times$ FPG $\left(\mathrm{mmol} \mathrm{I}^{-1}\right) / 22.5$. The index of $\beta$-cell function (homeostasis model assessment-pancreatic $\beta$-cell secretion $($ HOMA- $\beta))^{18}$ was calculated as follows: HOMA- $\beta=20 \times$ FINS $\left(\mathrm{mU} \mathrm{I}^{-1}\right) /$ (FPG $\left.\left(\mathrm{mmol}^{-1}\right)-3.5\right)$.

Measurement of body composition and abdominal fat distribution An automatic bioelectrical impedance analyzer (TBF-418B; Tanita Corp., Tokyo, Japan) was used to measure the total body fat mass (FM), fat percentage (fat\%) and free fat mass (FFM). The abdominal fat distribution, including the visceral fat area (VFA) and subcutaneous fat area (SFA), was assessed by magnetic resonance imaging (Archiva 3.0 T; Philips Medical Systems, Amsterdam, The Netherlands). The experienced examiner was aware of the study results. Image analysis software (slice-O-matic, version 4.2; Tomovision Inc., Montreal, Quebec, Canada) was used to calculate average VFA and SFA values according to a previously described protocol. $^{15}$

\section{Statistical analysis}

The SPSS 16.0 statistical software package (SPSS Inc., Chicago, IL, USA) was used for statistical analysis. The normality of the data distribution was determined by the one-sample Kolmogorov-Smirnov test. Data were expressed as mean \pm standard deviation or median with interquartile range according to a normal or skewed distribution, respectively. Comparisons between the two groups were carried out by an unpaired Student's $t$-test for normally distributed variables or the Mann-Whitney U-test for variables with a skewed distribution, and the $x^{2}$ test was used for categorical variables. Spearman's correlation coefficient analyses were conducted to assess the relationships of serum A-FABP levels with indexes of body fat and other metabolic parameters. Logistic regression analysis was conducted to uncover the relationship between a first-degree FHD and serum A-FABP levels. Multiple stepwise regression analysis was performed to identify the independent factor affecting serum A-FABP levels. The threshold of statistical significance was set at 0.05 for two-tailed $P$-values.

\section{RESULTS}

Clinical characteristics of study participants

A total of 1962 normoglycemic individuals were enrolled in the present study (age range: 20-78 years, median 51.94 (45.35-57.29) years), including 792 men (140 subjects with a first-degree FHD and 652 subjects without an FHD), 544 premenopausal women (114 subjects with a first-degree FHD and 430 subjects without an FHD) and 626 postmenopausal women (169 subjects with a first-degree FHD and 457 subjects without an FHD). In men, premenopausal women and postmenopausal women, subjects with a first-degree FHD had greater BMI, VFA and HOMA-IR values than those without an FHD in corresponding subgroups (all $P<0.05$ ). Additionally, subjects with a first-degree FHD had higher levels of FM, fat\%, FPG, HbA1c, FINS, HOMA- $\beta$, LDL-C and CRP than those without an FHD (all $P<0.05$ ) in men. Among premenopausal women, subjects with a first-degree FHD were older and exhibited higher levels of $\mathrm{FM}$, fat $\%, \mathrm{FPG}, \mathrm{TG}$ and LDL-c than individuals without an FHD (all $P<0.05$ ). Among postmenopausal women, the levels of $\mathrm{HbA} 1 \mathrm{c}$ and FINS were much higher in subjects with a first-degree FHD than those in individuals without an FHD (both $P<0.05$ ). Individuals with and without a first-degree FHD did not differ significantly with respect to other variables (all $P>0.05$; Table 1 ).

Serum A-FABP levels in participants with and without a firstdegree FHD

Among the entire study population, serum A-FABP levels were much higher in subjects with a first-degree FHD than in those without an FHD (4.27 (2.75-6.47) versus 3.63 (2.37-5.25) $\mathrm{ng} \mathrm{ml}^{-1}$; $P<0.001)$. Serum A-FABP levels increased in the order from men to premenopausal women to postmenopausal women (2.87 (1.93-4.33) versus $3.68(2.33-5.44) \mathrm{ng} \mathrm{ml}^{-1}$ and 3.68 $(2.33-5.44)$ versus $4.99(3.60-6.57) \mathrm{ng} \mathrm{m}^{1-1}$; both $\left.P<0.001\right)$. Comparisons conducted separately in men, premenopausal women and postmenopausal women confirmed the significant differences in serum A-FABP levels between individuals with and without a first-degree FHD ( $P=0.015,0.004$ and 0.029 , respectively; Figure 1).

Relationship between serum A-FABP levels and a first-degree FHD In men, premenopausal women and postmenopausal women, serum A-FABP levels were associated positively with indexes of body fat (body weight, FM, fat\%, FFM, VFA and SFA), HOMA-IR, HOMA- $\beta$, TG, LDL-C and CRP (all $P<0.05$ ), but negatively with $\mathrm{HDL}-\mathrm{c}$ (all $P<0.001$ ). Additional detailed results are displayed in Table 2

Logistic regression analysis revealed a positive relationship between a first-degree FHD and serum A-FABP levels in men $(P=0.001)$, premenopausal women $(P=0.001)$ and postmenopausal women $(P<0.001)$. After adjustment of the data for BMI, VFA, SFA and HOMA-IR separately, the positive association between a first-degree FHD and the serum A-FABP levels remained significant (all $P<0.05$ ). Taking into consideration age, BMI, W, VFA, SFA, systolic BP, diastolic BP, HbA1c, HOMA-IR, HOMA- $\beta$, TG, HDL-c, LDL-C and CRP for adjustment, multivariate logistic regression analysis further confirmed that a first-degree FHD was correlated with serum A-FABP levels both positively and independently $(P=0.029,0.036$ and 0.008 for men, premenopausal women, and postmenopausal women, respectively; Table 3).

Multiple stepwise regression analysis of serum A-FABP levels Upon defining the serum A-FABP levels as a dependent variable, multiple regression analysis took into account variables including a first-degree FHD, age, BMI, W, VFA, SFA, HbA1c, HOMA-IR, TG, HDL-C, LDL-C and CRP as independent variables. The results showed that a first-degree FHD was an independent factor positively associated with the serum A-FABP levels in men 


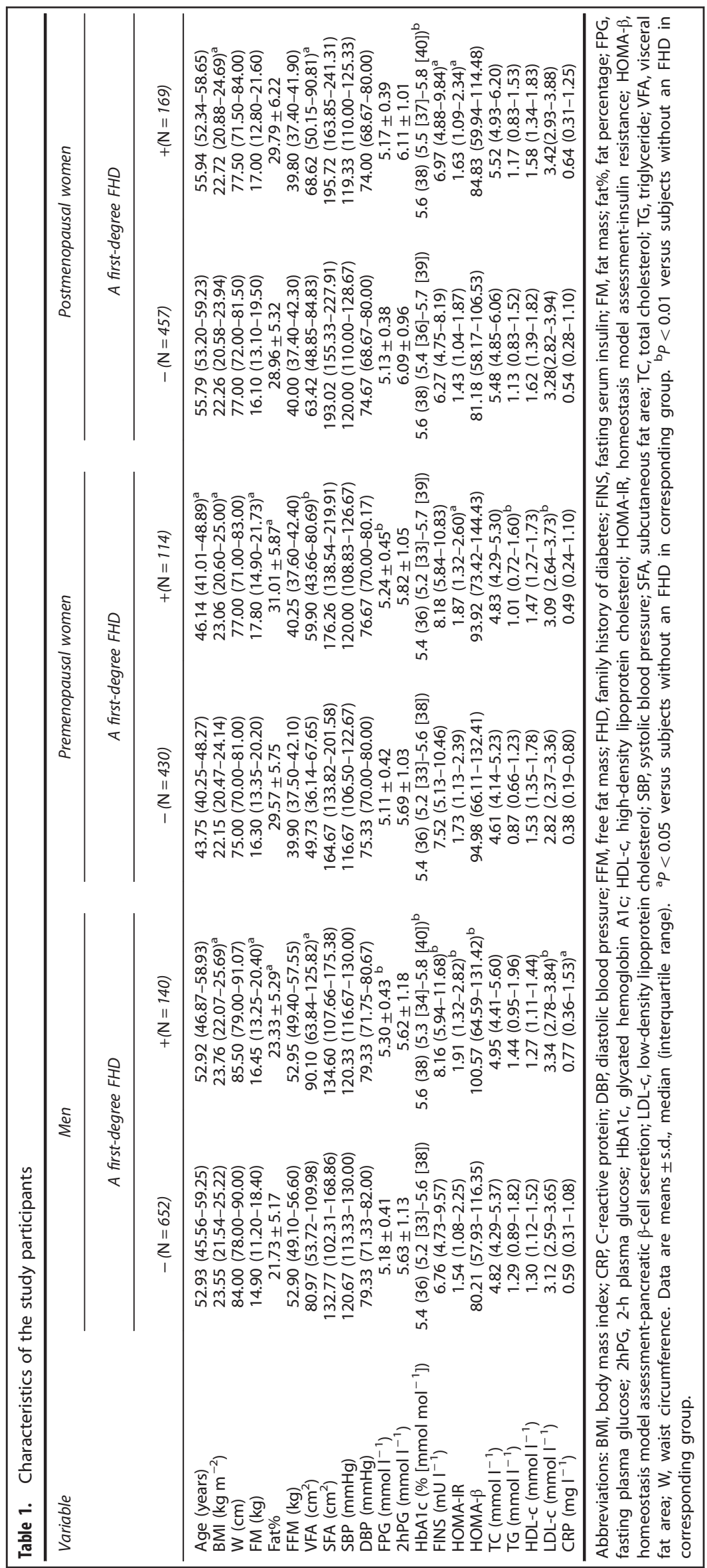




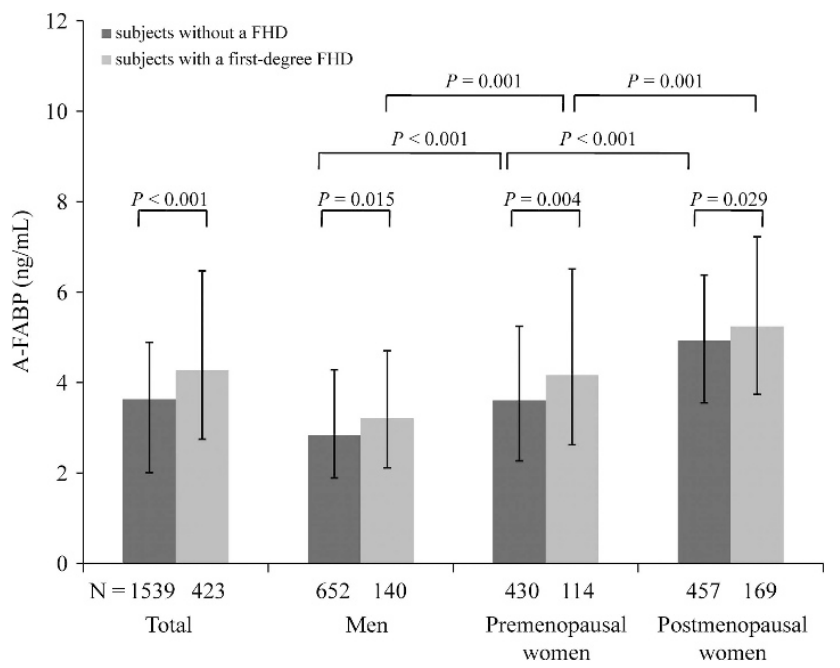

Figure 1. Subgroup comparisons of serum A-FABP levels between subjects with a first-degree FHD and those without an FHD. Serum A-FABP levels are expressed as median values with interquartile ranges.

\begin{tabular}{|c|c|c|c|c|c|c|}
\hline \multirow[t]{2}{*}{ Variable } & \multicolumn{2}{|c|}{ Men } & \multicolumn{2}{|c|}{$\begin{array}{c}\text { Premenopausal } \\
\text { women }\end{array}$} & \multicolumn{2}{|c|}{$\begin{array}{c}\text { Postmenopausal } \\
\text { women }\end{array}$} \\
\hline & $\mathrm{r}$ & $\mathrm{P}$ & $r$ & $\mathrm{P}$ & $r$ & $\mathrm{P}$ \\
\hline Body weight & 0.398 & $<0.001$ & 0.413 & $<0.001$ & 0.383 & $<0.001$ \\
\hline $\mathrm{FM}$ & 0.448 & $<0.001$ & 0.470 & $<0.001$ & 0.432 & $<0.001$ \\
\hline fat $\%$ & 0.430 & $<0.001$ & 0.459 & $<0.001$ & 0.416 & $<0.001$ \\
\hline FFM & 0.268 & $<0.001$ & 0.199 & $<0.001$ & 0.195 & $<0.001$ \\
\hline VFA & 0.449 & $<0.001$ & 0.406 & $<0.001$ & 0.378 & $<0.001$ \\
\hline SFA & 0.415 & $<0.001$ & 0.362 & $<0.001$ & 0.283 & $<0.001$ \\
\hline FPG & 0.070 & 0.047 & 0.062 & 0.147 & 0.057 & 0.154 \\
\hline 2hPG & 0.096 & 0.007 & 0.081 & 0.059 & 0.093 & 0.019 \\
\hline $\mathrm{HbA} 1 \mathrm{c}$ & 0.010 & 0.789 & 0.095 & 0.027 & 0.114 & 0.004 \\
\hline HOMA-IR & 0.362 & $<0.001$ & 0.266 & $<0.001$ & 0.290 & $<0.001$ \\
\hline HOMA- $\beta$ & 0.331 & $<0.001$ & 0.239 & $<0.001$ & 0.263 & $<0.001$ \\
\hline TC & 0.061 & 0.087 & 0.091 & 0.034 & 0.097 & 0.015 \\
\hline TG & 0.343 & $<0.001$ & 0.257 & $<0.001$ & 0.269 & $<0.001$ \\
\hline HDL-c & -0.310 & $<0.001$ & -0.121 & 0.005 & -0.215 & $<0.001$ \\
\hline LDL-c & 0.105 & 0.003 & 0.169 & $<0.001$ & 0.095 & 0.017 \\
\hline CRP & 0.244 & $<0.001$ & 0.277 & $<0.001$ & 0.251 & $<0.001$ \\
\hline
\end{tabular}

Abbreviations: CRP, C-reactive protein; FFM, free fat mass; FM, fat mass; fat $\%$, fat percentage; FPG, fasting plasma glucose; HDL-C, high-density lipoprotein cholesterol; 2hPG, 2-h plasma glucose; HbA1c, glycated hemoglobin A1c; HOMA-IR, homeostasis model assessment-insulin resistance; HOMA- $\beta$, homeostasis model assessment-pancreatic $\beta$-cell secretion; LDL-c, low-density lipoprotein cholesterol; SFA, subcutaneous fat area; TC, total cholesterol; TG, triglyceride; VFA, visceral fat area.

(standardized $\beta=0.068, P=0.029$ ), premenopausal women (standardized $\beta=0.090, \quad P=0.018$ ) and postmenopausal women (standardized $\beta=0.102, P=0.004$; Table 4).

\section{DISCUSSION}

In the present study, individuals with a first-degree FHD exhibited higher serum A-FABP levels than those without an FHD. After adjustment for indexes of body fat, glucose and lipid metabolism, and other related factors, a positive correlation between the serum
A-FABP levels and a first-degree FHD remained significant. Moreover, a first-degree FHD was further identified as a factor positively and independently associated with serum A-FABP levels. Despite gender differences in serum A-FABP levels, the same association between the serum A-FABP levels and a first-degree FHD held true for men, premenopausal women and postmenopausal women.

Henninger et al. ${ }^{19}$ revealed that markers of adipose tissue cell hypertrophy and dysfunction showed altered trends prior to the development of impaired glucose tolerance or T2DM in individuals with a first-degree FHD. Excess as well as suppressed secretion of adipokines are typical manifestations of adipocyte dysfunction. $^{20}$ Compared with other novel adipokines, the circulating A-FABP levels not only were higher, allowing for a visual representation of adipocyte-related metabolism, ${ }^{11}$ but also better predicted the onset of metabolic syndrome. ${ }^{21}$ Multiple clinical studies have reported that changes in serum levels of adipokines are not only present in individuals with a first-degree FHD, but also independently associated with a first-degree FHD. ${ }^{7-9}$ However, whether this is true for A-FABP remained to be determined. A previous study in a Chinese population only observed that serum A-FABP levels were higher in individuals with impaired glucose tolerance or impaired fasting glucose, and could predict the development of T2DM. ${ }^{22}$ The present study revealed for the first time a significant increase in serum A-FABP levels in normoglycemic individuals with a first-degree FHD, supporting the concept that adipocyte dysfunction appeared before the occurrence of glucose metabolism abnormalities in individuals with a first-degree FHD.

In a study including 8749 men without diabetes, Cederberg et al. $^{4}$ demonstrated that individuals with a first-degree FHD tended to present with overweight/obesity, particularly abdominal obesity. For this population, visceral fat, rather than subcutaneous fat, was a marker of dysfunctional adipose tissue and contributed to negative metabolic consequences. In our previous study, serum A-FABP levels were not only associated with FM but also influenced by the VFA in postmenopausal women. ${ }^{23}$ Simón et al. ${ }^{12}$ carried out a follow-up study in 77 women with morbid obesity and showed that at 1 year after bariatric surgery, circulating A-FABP levels decreased dramatically in parallel with massive weight loss, and these changes were accompanied by improvements in insulin sensitivity. These findings supported the role of A-FABP in obesity-induced insulin resistance. Consistent with these previous findings, the present study demonstrated that individuals with a first-degree FHD were more likely to have a greater body fat content, especially visceral fat. Serum A-FABP levels were related to total body fat content and abdominal fat distribution, as well as insulin resistance. Based on these clinical associations, we suggested that the elevated serum A-FABP levels in individuals with a first-degree FHD could be attributed, in part, to a susceptibility to increased total body fat content and visceral fat accumulation, and could further indicated insulin resistance.

Importantly, even after adjustment for body fat indexes, HOMA$I R$, and other variables related to glucose and lipid metabolism, serum A-FABP levels remained positively and significantly correlated with a first-degree FHD. Multivariate analysis identified a first-degree $\mathrm{FHD}$ as an independent factor contributing to elevated serum A-FABP levels. It followed that determinants, which induced an increase in serum A-FABP levels in individuals with a first-degree FHD, were not limited to the total body fat content and abdominal fat distribution. Clinical application of A-FABP has been investigated in several prospective and interventional studies. The circulating A-FABP levels were proposed as not only a predictor of the risk of metabolic and vascular disease but also an indicator for evaluating the beneficial effects of glucose control and other interventions such as weight reduction and statin use. ${ }^{24,25}$ In addition, researchers in the US provided a feasible approach for treating diabetes by targeting serum A-FABP with a monoclonal antibody (CA33). ${ }^{26}$ In light of 
Table 3. Association between a first-degree FHD and the serum A-FABP levels

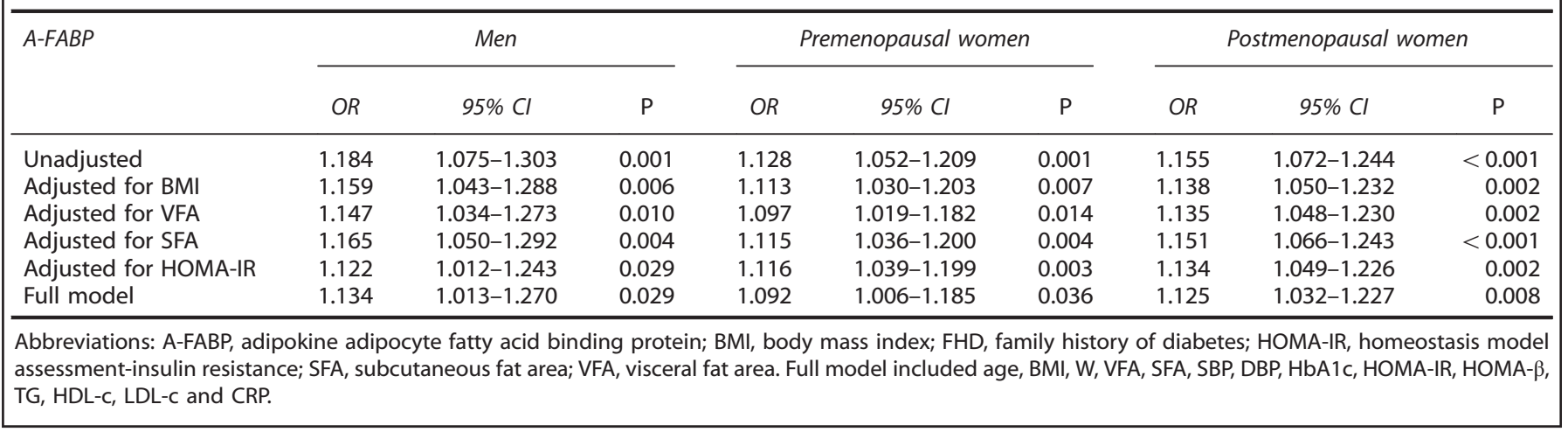

\begin{tabular}{|c|c|c|c|c|c|c|c|c|c|c|c|}
\hline $\begin{array}{l}\text { A first-degree } \\
\text { FHD }\end{array}$ & 0.068 & 2.184 & 0.029 & $\begin{array}{c}\text { A first-degree } \\
\text { FHD }\end{array}$ & 0.090 & 2.368 & 0.018 & $\begin{array}{c}\text { A first-degree } \\
\text { FHD }\end{array}$ & 0.102 & 2.866 & 0.004 \\
\hline SFA & 0.116 & 2.356 & 0.019 & HOMA-IR & 0.122 & 3.076 & 0.002 & HOMA-IR & 0.100 & 2.459 & 0.014 \\
\hline HOMA-IR & 0.136 & 3.676 & $<0.001$ & & & & & TG & 0.158 & 3.994 & $<0.001$ \\
\hline TG & 0.170 & 5.323 & $<0.001$ & & & & & & & & \\
\hline
\end{tabular}

this possibility of using the serum A-FABP levels in screening, diagnosis and treatment of diabetes in the clinical setting, it is necessary to consider the influence of a first-degree FHD on serum A-FABP levels. Negative metabolic effects and the risk of CVD could be overestimated according to the serum A-FABP levels if the individuals' FHD was not considered.

The first description of circulating A-FABP noted the gender difference. ${ }^{11}$ Subsequently, our previous study suggested that androgen contributes to the gender dimorphism of serum A-FABP levels through gender-specific effects on fat content and distribution. Furthermore, the effects of testosterone vary with the different active forms related to sex hormone-binding globulin between premenopausal women and postmenopausal women. ${ }^{17}$ Consistent with these findings, the present study confirmed that the serum A-FABP levels increased in the order from men to premenopausal women to postmenopausal women, suggesting that the gender- and menopause-related differences in serum A-FABP levels should be taken into consideration when A-FABP was used as a serum biomarker.

The potential mechanisms underlying the contribution of a firstdegree FHD to elevated serum A-FABP levels remained to be determined. Until now, there has been no direct evidence supporting the contribution of genetic factors to the alteration in serum A-FABP levels. However, individuals with a first-degree FHD inherit the susceptibility to impaired insulin action, which further develops epigenetically and leads to the increase in serum A-FABP levels. Cardellini et al. ${ }^{27}$ discovered that protein expression of insulin receptor substrate 2 and mRNA expression of tissue inhibitor of metalloproteinase 3 were decreased in first-degree relatives of patients with diabetes, representing the inhibition of the insulin signal transduction pathways and genetic modification of insulin action in this population. Additionally, in normoglycemic but insulin-resistant offspring of parents with T2DM, insulinstimulated AKT phosphorylation on $\mathrm{Ser}^{473}$ was suppressed, resulting in an approximately $60 \%$ reduction in AKT activation. ${ }^{28}$ Insulin could not only increase phosphorylation of AKT on $\mathrm{Ser}^{473}$ in a dosedependent manner ${ }^{29}$ to prevent forkhead box transcription factor 1 from promoting A-FABP gene transcription, ${ }^{30}$ but also downregulate $A-F A B P$ secretion from fat cells totally or partially via adipocyte-derived microvesicles. ${ }^{31}$ Therefore, impaired insulin action may be responsible for the increased serum A-FABP levels in individuals with a first-degree FHD.

There are some limitations in the present study. First, the crosssectional study design precluded the ability to determine a causal relationship between the serum A-FABP levels and insulin resistance. Second, the influence of genetic predisposition on serum A-FABP levels remained unclear. The notion that genetic factors were associated with elevated serum A-FABP levels must be further validated in basic research studies.

In conclusion, serum A-FABP levels were significantly greater in individuals with a first-degree FHD than in those without an FHD even among individuals with normal blood glucose levels. The contribution of a first-degree FHD to the elevated serum A-FABP levels was independent of the total body fat content and abdominal fat distribution. Thus, in individuals with a firstdegree FHD, use of the serum A-FABP levels as a biomarker for increased risk of obesity-induced metabolic disease and CVD might result in overestimation of these risks. In this case, an FHD should not be ignored when interpreting elevated serum A-FABP levels in the clinical setting. 


\section{CONFLICT OF INTEREST}

The authors declare no conflict of interest.

\section{ACKNOWLEDGEMENTS}

This work was funded by 973 Program of China (2013CB530606), grant from Shanghai Health and Family Planning Commission (2013ZYJB1001), Project of National Natural Science Foundation of China (81100563) and Translational Medicine Innovation Foundation of School of Medicine Shanghai Jiao Tong University (15ZH2010 and 15ZH4006).

\section{REFERENCES}

1 Wagner R, Thorand B, Osterhoff MA, Müller G, Böhm A, Meisinger C et al. Family history of diabetes is associated with higher risk for prediabetes: a multicentre analysis from the German Center for Diabetes Research. Diabetologia 2013; 56: 2176-2180.

2 Perseghin G, Ghosh S, Gerow K, Shulman Gl. Metabolic defects in lean nondiabetic offspring of NIDDM parents: a crosssectional study. Diabetes 1997; 46: 1001-1009.

3 Wilson PWF, Meigs JB, Sullivan L, Fox CS, Nathan DM, D'Agostino RB Sr. Prediction of incident diabetes mellitus in middle-aged adults: the Framingham Offspring Study. Arch Intern Med 2007; 167: 1068-1074.

4 Cederberg H, Stančáková A, Kuusisto J, Laakso M, Smith U. Family history of type 2 diabetes increases the risk of both obesity and its complications: is type 2 diabetes a disease of inappropriate lipid storage? J Intern Med 2015; 277: 540-551.

5 Stadler M, Pacini G, Petrie J, Luger A, Anderwald C, RISC Investigators. Beta cell (dys)function in non-diabetic offspring of diabetic patients. Diabetologia 2009; 52 : 2435-2444.

6 Goldfine AB, Beckman JA, Betensky RA, Devlin H, Hurley S, Varo N et al. Family history of diabetes is a major determinant of endothelial function. $J$ Am Coll Cardiol 2006; 47: 2456-2461.

7 Akbarzadeh S, Nabipour I, Assadi M, Movahed A, Jafari SM, Motamed N et al. The normoglycemic first-degree relatives of patients with type 2 diabetes mellitus have low circulating omentin-1 and adiponectin levels. Cytokine 2012; 58: 295-299.

8 Akbarzadeh S, Nabipour I, Jafari SM, Movahed A, Motamed N, Assadi M et al. Serum visfatin and vaspin levels in normoglycemic first-degree relatives of Iranian patients with type 2 diabetes mellitus. Diabetes Res Clin Pract 2012; 95: 132-138.

9 Bose KS, Gupta SK, Singh S. Is serum retinol binding protein-4: a predictor for diabetes in genetically high risk population? J Res Med Sci 2012; 17: 1015-1019.

10 Chmurzyńska A. The multigene family of fatty acid-binding proteins (FABPs): function, structure and polymorphism. J Appl Genet 2006; 47: 39-48.

11 Xu A, Wang Y, Xu JY, Stejskal D, Tam S, Zhang J et al. Adipocyte fatty acid-binding protein is a plasma biomarker closely associated with obesity and metabolic syndrome. Clin Chem 2006; 52: 405-413.

12 Simón I, Escoté X, Vilarrasa N, Gómez J, Fernández-Real JM, Megía A et al. Adipocyte fatty acid-binding protein as a determinant of insulin sensitivity in morbid-obese women. Obesity (Silver Spring) 2009; 17: 1124-1128.

13 Hao Y, Ma X, Luo Y, Shen Y, Dou J, Pan X et al. Serum adipocyte fatty acid binding protein levels are positively associated with subclinical atherosclerosis in Chinese pre- and postmenopausal women with normal glucose tolerance. J Clin Endocrinol Metab 2014; 99: 4321-4327.

14 Zhou M, Bao Y, Li H, Pan Y, Shu L, Xia Z et al. Deficiency of adipocyte fatty-acidbinding protein alleviates myocardial ischaemia/reperfusion injury and diabetesinduced cardiac dysfunction. Clin Sci (Lond) 2015; 129: 547-559.

15 Bao Y, Ma X, Yang R, Wang F, Hao Y, Dou J et al. Inverse relationship between serum osteocalcin levels and visceral fat area in Chinese men. $J$ Clin Endocrinol Metab 2013; 98: 345-351.

16 World Health Organization. Department of Noncommunicable Disease Surveillance. Definition, diagnosis and classification of diabetes mellitus and its complication. Report of a WHO consultation. Part 1: Diagnosis and classification of diabetes mellitus. World Health Organization: Geneva, 1999.
17 Hu X, Ma X, Pan X, Luo Y, Xu Y, Xiong Q et al. Association of androgen with gender difference in serum adipocyte fatty acid binding protein levels. Sci Rep 2016; 6: 27762.

18 Matthews DR, Hosker JP, Rudenski AS, Naylor BA, Treacher DF, Turner RC. Homeostasis model assessment: insulin resistance and beta-cell function from fasting plasma glucose and insulin concentrations in man. Diabetologia 1985; 28: 412-419.

19 Henninger J, Hammarstedt A, Rawshani A, Eliasson B. Metabolic predictors of impaired glucose tolerance and type 2 diabetes in a predisposed population-a prospective cohort study. BMC Endocr Disord 2015; 15: 51.

20 Gustafson B, Gogg S, Hedjazifar S, Jenndahl L, Hammarstedt A, Smith U. Inflammation and impaired adipogenesis in hypertrophic obesity in man. Am J Physiol Endocrinol Metab 2009; 297: E999-E1003.

21 Choi KM, Yannakoulia M, Park MS, Cho GJ, Kim JH, Lee SH et al. Serum adipocyte fatty acid-binding protein, retinol-binding protein 4 , and adiponectin concentrations in relation to the development of the metabolic syndrome in Korean boys: a 3-y prospective cohort study. Am J Clin Nutr 2011; 93: 19-26.

22 Tso AW, Xu A, Sham PC, Wat NM, Wang Y, Fong CH et al. Serum adipocyte fatty acid binding protein as a new biomarker predicting the development of type 2 diabetes: a 10-year prospective study in a Chinese cohort. Diabetes Care 2007; 30: 2667-2672.

23 Hao Y, Ma X, Luo Y, Hu X, Pan X, Xiao Y et al. Associations of serum adipocyte fatty acid binding protein with body composition and fat distribution in nondiabetic Chinese women. J Clin Endocrinol Metab 2015; 100: 2055-2062.

24 Kralisch S, Fasshauer M. Adipocyte fatty acid binding protein: a novel adipokine involved in the pathogenesis of metabolic and vascular disease? Diabetologia 2013; 56: 10-21.

25 Na LX, Yan BL, Jiang S, Cui HL, Li Y, Sun CH. Curcuminoids target decreasing serum adipocyte-fatty acid binding protein levels in their glucose-lowering effect in patients with type 2 diabetes. Biomed Environ Sci 2014; 27: 902-906.

26 Burak MF, Inouye KE, White A, Lee A, Tuncman G, Calay ES et al. Development of a therapeutic monoclonal antibody that targets secreted fatty acid-binding protein aP2 to treat type 2 diabetes. Sci Transl Med 2015; 7: 319ra205.

27 Cardellini M, Menghini R, Luzi A, Davato F, Cardolini I, D'Alfonso R et al. Decreased IRS2 and TIMP3 expression in monocytes from offspring of type 2 diabetic patients is correlated with insulin resistance and increased intima-media thickness. Diabetes 2011; 60: 3265-3270.

28 Morino K, Petersen KF, Dufour S, Befroy D, Frattini J, Shatzkes N et al. Reduced mitochondrial density and increased IRS-1 serine phosphorylation in muscle of insulin-resistant offspring of type 2 diabetic parents. J Clin Invest 2005; 115: 3587-3593.

29 Karlsson HK, Ahlsén M, Zierath JR, Wallberg-Henriksson H, Koistinen HA. Insulin signaling and glucose transport in skeletal muscle from first-degree relatives of type 2 diabetic patients. Diabetes 2006; 55: 1283-1288.

30 Harjes U, Bridges E, Mclntyre A, Fielding BA, Harris AL. Fatty acid-binding protein 4 , a point of convergence for angiogenic and metabolic signaling pathways in endothelial cells. J Biol Chem 2014; 289: 23168-23176.

31 Kralisch S, Ebert T, Lossner U, Jessnitzer B, Stumvoll M, Fasshauer M. Adipocyte fatty acid-binding protein is released from adipocytes by a non-conventional mechanism. Int J Obes (Lond) 2014; 38: 1251-1254.

(c) $\Theta$ This work is licensed under a Creative Commons Attributioncc) NonCommercial-NoDerivs 4.0 International License. The images or other third party material in this article are included in the article's Creative Commons license, unless indicated otherwise in the credit line; if the material is not included under the Creative Commons license, users will need to obtain permission from the license holder to reproduce the material. To view a copy of this license, visit http:// creativecommons.org/licenses/by-nc-nd/4.0/

(c) The Author(s) 2016 